\title{
AA.VV.
}

\section{LINGUAGGI DELL'ORGANIZZARE}

Musica e testo tra dono e disinteresse

\author{
Luigi Maria Sicca \\ a cura di
}

Editoriale Scientifica

Napoli 
Il volume è stato finanziato dal Dipartimento di Economia Aziendale, Università degli Studi di Napoli Federico II.

Tutti i diritti sono riservati

I testi degli Haiku sono di Vincenzo Capitanucci, Liana Margescu e Augusto Villa che hanno esplicitamente autorizzato Lorenzo Pone, autore del capitolo "Quattro Haiku per voce, violoncello e pianoforte op. 4 ", ad adottarli per la pubblicazione del presente volume.

(C) 2013 Editoriale Scientifica srl

Via San Biagio dei Librai 39

80138 Napoli

www.editorialescientifica.com

info@editorialescientifica.com

ISBN 978-88-6342-477-5 


\section{Indice}

$7 \quad$ LE RAGIONI DI UNA RICERCA

Luigi Maria Sicca

31 APPUNTI DI NEUROBIOLOGIA DEL SUONO E DEL LINGUAGGIO Umberto di Porzio

49 IL DONO IN CONCERTO. UNA RIFLESSIONE SUL PLUSVALORE DONATIVO NEL LAVORO DI UN SOLISTA Rosario Diana

65 IL SUONO COME DONO NON DISINTERESSATO. SPUNTI PER UNA BIOPOLITICA DELLA MUSICA Agostino Di Scipio

85 LA "MUSA MUNIFICA": DAL “DIVINO DONO" DELL'AEDO ALLA RETORICA DEL MUSICISTA-BENEFATTORE Mariella De Simone

105 PRÀXIS

Bernardo Maria Sannino

123 TO-TEM N ${ }^{\circ} 2$

Chiara Mallozzi

137 QuatTro HaIkU OP. 4 Lorenzo Pone

157 IL DONO DEL SUONO.

RIFLESSIONI PER UNA POIETICA DELL'ASCOLTO

Giancarlo Turaccio

185 Indice dei nomi

189 Notizie sugli Autori 